\title{
Why do disordered and structured proteins behave differently in phase separation?
}

\author{
Huan-Xiang Zhou ${ }^{1,2,}{ }^{*}$, Valery Nguemaha ${ }^{2}$, Konstantinos Mazarakos ${ }^{2}$, and Sanbo Qin ${ }^{2}$ \\ ${ }^{1}$ Department of Chemistry and Department of Physics, University of Illinois at Chicago, Chicago, \\ IL 60607, USA \\ 2Department of Physics and Institute of Molecular Biophysics, Florida State University, \\ Tallahassee, FL 32306, USA
}

\begin{abstract}
Intracellular membraneless organelles and their myriad cellular functions have garnered tremendous recent interest. It is becoming well accepted that they form via liquid-liquid phase separation (LLPS) of protein mixtures (often including RNA), where the organelles correspond to a protein-rich, droplet phase coexisting with a protein-poor, bulk phase. The major protein components contain disordered regions and often also RNA-binding domains, and the disordered fragments on their own easily undergo LLPS. In contrast, LLPS for structured proteins has been observed infrequently. The contrasting phase behaviors can be explained by modeling disordered and structured proteins, respectively, as polymers and colloids. These physical models also provide better understanding on the regulation of droplet formation by cellular signals and dysregulation leading to diseases.
\end{abstract}

\section{Keywords}

Membarneless organelle; phase separation; transient bonding network

\section{MLOs: disordered proteins as drivers and myriad cellular functions}

\begin{abstract}
Whereas the roles of most membrane-bound organelles have been established over a century, characterization of the molecular and physical features of membraneless organelles (MLOs; see Glossary) has started only in recent years. Already, they have been associated with a wide range of biological functions, including RNA processing, ribosome biogenesis, and sequestration of mRNA (for later translation), proteins (for signaling), and compacted chromatin (for gene silencing) [1-3]. It is now well accepted that MLOs form via liquidliquid phase separation (LLPS; Box 1), whereby they emerge from the cytoplasm or nucleoplasm as protein-rich droplets [4-8]. Like their membrane-bound counterparts, each
\end{abstract}

\footnotetext{
*Corresponding author: Zhou, H.-X. (hzhou43@uic.edu).

Publisher's Disclaimer: This is a PDF file of an unedited manuscript that has been accepted for publication. As a service to our customers we are providing this early version of the manuscript. The manuscript will undergo copyediting, typesetting, and review of the resulting proof before it is published in its final citable form. Please note that during the production process errors may be discovered which could affect the content, and all legal disclaimers that apply to the journal pertain.
} 
type of MLO contains many different species of macromolecules, but the MLO identity can often be associated with one or more "driver" proteins that are most responsible for the assembly and function of the organelle [9]. For example, fibrillarin (FIB1) and nucleophosmin (NPM1) are putatively driver proteins for the subcompartmentalized organization of the nucleolus, an organelle with a primary function in ribosome preassembly [10]. In a number of better-characterized cases, the driver proteins always have extended, intrinsically disordered regions (IDRs), which sometimes also have RNA-binding ability (Figure 1a); quite often though, one or more separate RNA-binding domains, such as RNA-recognition motifs (RRMs), are present as well [11] (Table 1). Many studies with deletion constructs have shown that the IDRs are sufficient and perhaps necessary for LLPS [10, 12-23]. It has been speculated that intrinsic disorder may promote LLPS by enabling multiple, weakly attractive interactions $[12,14,17,24,25]$, but the mechanism largely remains unclear.

A hallmark of LLPS (in contrast to, e.g., protein aggregation) is thermodynamic reversibility, consistent with the liquid-like character of the droplet phase. That is, droplets can easily dissolve upon raising temperature or salt concentration and can reform when conditions are reverted. The reversibility comes because the two phases, droplet and dispersed, are in thermodynamic equilibrium (Box 1). Phase separation occurs when molecules can achieve the same low free energy by adopting two distinct types of configurations, with disparate concentrations and extents of intermolecular contacts [26]. The low free energy is achieved by distinct means in the two phases: e.g., high entropy (due to low concentration) in the dispersed phase but strong favorable (i.e., negative) enthalpy (from intermolecular contacts) in the droplet phase. Raising temperature or salt concentration leads to reduced stabilization of the droplet phase by intermolecular contacts. Beyond a critical point, the distinction between the two phases vanishes, and hence droplets dissolve. In general, the more strongly attractive the intermolecular interactions are, the wider the range of conditions for phase separation is (corresponding to raised critical temperature or critical salt concentration).

Synthetic polymers have long been known to undergo LLPS [27]. As with other thermodynamic properties of intrinsically disordered proteins (IDPs) [28], polymer models have been invoked to analyze phase boundaries. Specifically, the Flory-Huggins theory and its extensions (Box 2) have been used to fit experimental data or construct phase diagrams, providing explanations for the effects of salts, RNA, and sequence charge patterns on phase boundaries [17, 29-31]. Salt ions can screen charge-charge attraction between protein residues, and hence droplets dissolve above a critical salt concentration. Several studies have shown that the addition of RNA promoted LLPS, indicated by either reduced threshold protein concentrations for droplet formation or increased critical salt concentrations [5, 10, 14, 15, 21, 24, 32]. On the other hand, Zhang et al. [19] and Banerjee et al. [33] observed that RNA promoted LLPS only up to a point in RNA concentration, with further increase in RNA leading to LLPS suppression. In yet another variation, Wei et al. [29] recently found that, for LAF-1, a disordered driver protein for P granules [13], RNA had little effects on the threshold protein concentration and critical salt concentration, but significantly reduced the protein concentration in the droplet phase (Figure 1b). These conflicting reports were resolved in a recent theoretical study [34] (see below). 
Wei et al. [29] noted that the LAF-1 concentrations in the dissolved and droplet phases, approximately 0.1 and $5 \mathrm{mg} / \mathrm{ml}$, respectively, are orders of magnitude lower than the counterparts of structured proteins including lysozyme [35] and $\gamma$-crystallins (Figure 1c,d) [36]. This contrast buttresses the notion that LLPS occurs much more easily for disordered proteins than for structured proteins, which is a focus of the present review.

\section{LLPS of structured proteins: metastability relative to fluid-solid transition}

LLPS was actually first observed on structured proteins, as a metastable step on the way to crystallization [37, 38]. The metastability (Figure 1d) can be attributed to the small ratio between the range of attractive interactions and diameter of proteins, as demonstrated on spherical models of colloidal particles (Box 3). Kinetically, the metastable droplets rich in proteins can facilitate nucleation and thereby accelerate crystallization [39-42].

Under conditions for slow crystallization, the LLPS coexistence curves of several structured proteins have been determined, including arachin [37], lysozyme [35], $\gamma$-crystallins (Figure 1d) [36, 43], with critical protein concentrations ranged from 180 to $276 \mathrm{mg} / \mathrm{ml}$. The critical concentrations of immunoglobulin $\mathrm{G}$ ( $\mathrm{IgG}$ ) antibodies were somewhat lower, at approximately $90 \mathrm{mg} / \mathrm{ml}$, which was attributed to the nonspherical shape of the antibody molecules [44]. Recently a peptide oligomer was found to phase separate with an even lower critical concentration of $50 \mathrm{mg} / \mathrm{ml}$; loose packing within the oligomer was suggested as an explanation [45].

The high concentrations required pose one difficulty for observing LLPS of structured proteins. Structured proteins also tend to have low critical temperatures $\left(T_{\mathrm{c}}\right)$. When $T_{\mathrm{c}}$ is below the freezing point of the protein solution, it is not possible to directly observe LLPS. Crowding agents like polyethylene glycol (PEG) have been found to promote LLPS, raising $T_{\mathrm{c}}$ to above the freezing point $[43,45]$. By extrapolating, one can deduce the coexistence curve for the protein in the absence of crowding.

\section{Transient bonding networks: common organizational principle for protein droplets}

So far there has been very little crosstalk between the growing community of scientists working on LLPS of MLO-driving disordered proteins and the more established counterpart on LLPS of structured proteins. Connecting the research in the two communities will help us establish a common physical basis for phase separation of proteins, exchange knowledge between the two communities, and identify what are unique about IDPs and about structured proteins.

Droplet formation requires a sufficient extent of attraction between protein molecules to provide stability. On the other hand, to be in a liquid phase, individual interactions between two molecules must be easily breakable and hence relatively weak. Therefore, by necessity, proteins, whether structured or disordered, in the droplet phase form weakly attractive interactions with multiple partners, resulting in transient bonding networks (Figure 2a) [46]. 
For rigid structured proteins, it has become possible to use an all-atom representation in simulating phase separation and calculating coexistence curves (Figure 2) [46]. These simulations provide an atomistic view of the bonding networks in protein droplets. With atomistic calculations of coexistence curves, it is possible to test whether ideas such as nonspherical shape and loose packing are correct in explaining low critical concentrations for some structured proteins. Moreover, even highly homologous $\gamma$-crystallins can differ in $T_{\mathrm{c}}$ in excess of $30^{\circ} \mathrm{C}$ (Figure 1d). The calculations afford an opportunity to relate changes in phase behavior to changes in amino acid sequence. Such relations may provide valuable insight for understanding how mutations (e.g., ones associated with diseases) and posttranslational modifications affect LLPS of MLO-driving proteins (see below).

In addition to changes in amino acid sequence, the presence of other macromolecules can also influence the bonding networks of droplet-forming proteins and hence their phase behaviors. As noted, PEG has been used to raise $T_{\mathrm{c}}$ for structured proteins $[43,45]$. Similarly, crowding agents such as PEG, Ficoll, and dextran have been found to promote LLPS of MLO-driving proteins [14, 15, 47].

Crowders promote LLPS by preferentially partitioning in the dispersed phase, and therefore displacing proteins into the droplet phase to strengthen bonding networks there [34]. A macromolecular component, e.g., another protein or RNA, starts to partition in the droplet phase if it has attraction for the droplet-forming protein. When the macromolecule-protein attraction is mild, the presence of the macromolecule in the droplet phase serves to disrupt the bonding networks of the protein and thereby suppress LLPS. Such an effect was observed for human and bovine serum albumin, respectively, on the phase separation of an IgG antibody [44] and hnRNPA1 [48]. On the other hand, when the macromolecule-protein attraction is stronger than the protein-protein attraction, the macromolecule can strengthen the protein bonding networks and thus promote LLPS. This is likely the reason for the effects of RNA in several studies [5, 10, 14, 15, 21, 24, 32]. However, the promotional effects persist only up to a certain RNA-to-protein molar ratio, beyond which RNA displaces too much of the protein from the droplet phase and thereby again disrupts the bonding networks. Such dual effects of RNA have been observed by Zhang et al. [19] and Banerjee et al. [33].

It should be noted that MLO-driving proteins often contain both IDRs and structured domains (including those binding RNA; see Table 1). Several studies have shown that IDRs and structured domains can act synergistically in promoting phase separation [24, 48-50].

\section{Physical basis for contrasting phase behaviors of disordered and structured proteins}

A common physical basis notwithstanding, structured and disordered proteins nevertheless exhibit very different phase behaviors, in terms of high $T_{\mathrm{c}}$ and low critical concentrations for the latter proteins and the general ease in observing LLPS for them. Whereas structured proteins can form crystals and LLPS for them is often metastable relative to the fluid-solid transition, disordered proteins cannot form crystals and hence do not have a similar fluid- 
solid transition for competition [26]. Disordered proteins can form solid-like condensates such as gels and fibrils.

The contrast in $T_{\mathrm{c}}$ and critical concentration between structured and disordered proteins is reminiscent of the differing phase behaviors of colloid particles (Box 3) and polymer chains (Box 4). We represent colloid or polymer concentration by the volume fraction $\phi$. Relative to a colloid that models globular proteins, a 100-residue polymer, which could serve as a model for IDPs, has a $T_{\mathrm{c}}$ that is 4.2 times higher and a critical concentration $\left(\phi_{\mathrm{c}}\right)$ that is 5.1 times lower. How can we understand these differences?

At phase equilibrium, the dispersed and droplet phases must have equal chemical potentials. The chemical potential can be decomposed,

$$
\mu=\mu^{\mathrm{id}}+\mu^{\mathrm{rep}}+\mu^{\mathrm{att}}
$$

The first term on the right-hand side is the ideal part, which is the chemical potential if intermolecular interactions were totally absent, given by

$$
\mu^{\text {id }}=k_{B} T \ln \phi
$$

The second term, $\mu^{\text {rep }}$, is the contribution from steric repulsion between colloids or polymers; this would be the only contribution from intermolecular interactions if the molecules were purely repulsive toward each other. This term is positive and an increasing function of $\phi$, reflecting the difficulty in inserting a molecule into an already crowded solution. The third term, $\mu^{\text {att }}$, is the contribution from intermolecular interactions beyond steric repulsion, and does not have to be purely enthalpic. The $\mu^{\text {id }}$ and $\mu^{\text {rep }}$ terms favor the dispersed phase, whereas $\mu^{\text {att }}$ favors the droplet phase and must become sufficiently negative at increasing $\phi$ for LLPS to occur. (Note that, when crowders are present, they preferentially partition into the dispersed phase and raise $\mu^{\text {rep }}$ there; then $\mu^{\text {att }}$ does not need to be as negative as in the absence of crowders.) In general $T_{\mathrm{c}}$ increases with increasing magnitude of $\mu^{\text {att }}$.

In Figure 3, Key Figure we compare the component and total chemical potentials of the colloid and 100-residue polymer. Each monomer unit of the polymer can have attractive interactions with all other monomers (in the same chain or in other chains), in a way similar to attractive interactions between colloid particles. Therefore $\mu^{\text {att }}$ for the polymer accumulates over the monomer units, leading to a much higher $T_{\mathrm{c}}$ than that of the colloid. Due to the same reason, the chemical potential results for the polymer are shown in Figure $3 \mathrm{~b}$ on a per monomer basis (i.e., divided by the chain length $L$ ), and at a temperature that is 4 times higher than for the colloid. For the colloid, $\mu^{\text {id }}$ has a rapid rise near $\phi=0$, and subsequently, $\mu^{\text {rep }}$ takes off; $\mu^{\text {att }}$ catches up with $\mu^{\text {id }}+\mu^{\text {re }}$ p only at a high $\phi$. That explains why the concentrations of colloids (and, by inference, globular proteins) in the droplet phase are high. The concentrations of colloids in the dispersed phase cannot be too low either, 
since that would make the dispersed phase too stable (due to a strongly favorable $\mu^{\text {id }}$ ) compared with the droplet phase.

In contrast, for the polymer, on a per monomer basis, $\mu^{\mathrm{id}} / L$ is small, and the rise of $\mu^{\mathrm{rep}} / L$ is much less rapid than $\mu^{\text {rep }}$ for a colloid particle (the two would be the same were the

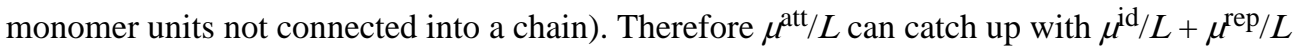
at relatively low $\phi$. That explains why the concentrations of polymers (and, by inference, IDPs) in the droplet phase are low.

The polymer model also predicts a much higher $T_{\mathrm{c}}$ than the colloid model (see Box 3 Figure I and Box 4 Figure I). This difference is related to the fact that each monomer unit in a polymer chain has the freedom to interact with all the monomer units in any other chain. Therefore the flexible polymer chains, as well as IDPs, can easily form multivalent interactions.

\section{Regulation of MLO formation by cellular signals}

The foregoing presentation makes it easy to explain how various cellular signals can regulate protein droplet formation. Environmental stress can be transmitted into changes in intracellular osmotic pressure or temperature [17, 51], and these basic physical changes can bring proteins into or out of LLPS conditions. Effects of varying salt concentrations and $\mathrm{pH}$ on LLPS, by modulating the magnitude of $\mu^{\text {att }}$, have been amply demonstrated in in vitro studies, and are likely exploited in vivo. Interestingly, cells may also perturb $\mu^{\text {rep }}$, by changing the level of macromolecular crowding through an increase or decrease in cell size [52].

LLPS occurs below the critical point but only when protein concentrations are above a threshold. Therefore intracellular protein concentration provides another means for regulating MLO formation [15]. RNA in particular can promote phase separation, but only within a certain range, and hence RNA expression level can also control whether MLOs form. Molecules that compete for RNA binding with droplet-forming proteins can limit the amount of RNA that enter into droplets and thus provide yet another layer of regulation [21, 32].

Posttranslational modifications (PTMs) regulate molecular structural organizations at all levels, and MLOs are no exception. The IDRs of droplet-forming proteins provide ready accessibility for PTMs, and the PTMs can promote or suppress LLPS by enabling or disrupting intermolecular interactions. For example, Tyr phosphorylations of the nephrin Cterminal IDR enable multivalent binding with Nck SH2 domains, and thereby promote LLPS of the Nck/N-WASP system [5]. On the other hand, Ser/Thr phosphorylation and phosphomimetic (Ser/Thr to Glu) mutation of the FUS N-terminal IDR disrupt transient intermolecular interactions and suppress LLPS [53]. Similarly, Ser phosphorylation of the MEG group of IDPs promotes P granule disassembly whereas dephosphorylation promotes granule assembly [54]. Arg methylations of both the hnRNPA2 C-terminal IDR [23] and the DDX4 N-terminal IDR [17] were found to suppress LLPS. An alternative splicing of the DDX4 N-terminal IDR also abrogated LLPS [17]. 
Droplets of structured proteins can nucleate crystal growth and have been associated with the genesis of protein condensation diseases such as cataract $[38,55,56]$ and sickle cell anemia [57, 58]. Similarly, LLPS of disordered proteins can facilitate further transition to solid-like condensates such as gels and fibrils, either as normal cellular response [59] or for pathogenesis [15, 22, 23, 47, 53, 60]. Disease-associated mutations may either promote LLPS or accelerate liquid-to-solid transition.

\section{Concluding remarks}

The physical basis for the LLPS of proteins, both structured and disordered, is becoming clear. Proteins are driven into the dispersed and droplet phases by different forces: translational freedom of the individual molecules and steric repulsion between them for the dispersed phase whereas intermolecular attraction for the droplet phase. It is important to recognize that the phase equilibrium is determined by the balance of steric (or crowding) and attractive interactions. Macromolecular crowders, by preferentially partitioning into the dispersed phase, can have significant effects on LLPS, as demonstrated in computational and in vitro experimental studies, and very likely on in vivo MLO formation. Other macromolecules, in particular RNA, can partition into the droplet phase, thereby strengthening or disrupting transient bonding networks there and affecting the phase equilibrium.

Macromolecular crowders and RNA are respective examples of species that are excluded from and recruited into protein droplets. MLOs contain multiple components and are surrounded by many more non-component species. Moreover, different types of MLOs may be immiscible and may even organize into core-shell structures (droplet inside droplet) [10]. A fruitful direction would be to characterize the differential partitioning of various species and their effects on MLO assembly and disassembly (see Outstanding Questions).

Our knowledge about transient bonding networks inside protein droplets is still scant. Here computation can well complement structural biology techniques. For structured proteins, computation at the all-atom level is already feasible [46], but for disordered proteins, conformational flexibility has limited computation to a coarsegrained level [23]. For subtle effects of PTMs and disease-causing mutations, an all-atom representation may be necessary for quantitative prediction. All-atom computation for IDP LLPS will be a great challenge for the future.

Transitions of IDP droplets into solid-like condensates (e.g., gels and fibrils) are implicated both in normal cellular responses and in diseases. A general physical model for such transitions is yet to be developed, in part because they often appear irreversible. Once again one may have to go to the colloid and polymer literature for some guidance.

\section{Acknowledgments}

This work was supported by National Institutes of Health Grant GM118091. 


\section{References}

1. Mitrea DM, Kriwacki RW. Phase separation in biology; functional organization of a higher order. Cell Commun Signal. 2016; 14:1. [PubMed: 26727894]

2. Larson AG, et al. Liquid droplet formation by HP1alpha suggests a role for phase separation in heterochromatin. Nature. 2017; 547:236-240. [PubMed: 28636604]

3. Strom AR, et al. Phase separation drives heterochromatin domain formation. Nature. 2017; 547:241-245. [PubMed: 28636597]

4. Brangwynne $\mathrm{CP}$, et al. Germline $\mathrm{P}$ granules are liquid droplets that localize by controlled dissolution/condensation. Science. 2009; 324:1729-1732. [PubMed: 19460965]

5. Li P, et al. Phase transitions in the assembly of multivalent signalling proteins. Nature. 2012; 483:336-340. [PubMed: 22398450]

6. Hyman AA, et al. Liquid-liquid phase separation in biology. Annu Rev Cell Dev Biol. 2014; 30:3958. [PubMed: 25288112]

7. Brangwynne CP, et al. Polymer physics of intracellular phase transitions. Nat Phys. 2015; 11:899904.

8. Qin S, Zhou HX. Protein folding, binding, and droplet formation in cell-like conditions. Curr Opin Struct Biol. 2017; 43:28-37. [PubMed: 27771543]

9. Aguilera-Gomez A, Rabouille C. Membrane-bound organelles versus membrane-less compartments and their control of anabolic pathways in Drosophila. Dev Biol. 2017; 428:310-317. [PubMed: 28377034]

10. Feric M, et al. Coexisting liquid phases underlie nucleolar subcompartments. Cell. 2016; 165:1686-1697. [PubMed: 27212236]

11. Courchaine EM, et al. Droplet organelles? EMBO J. 2016; 35:1603-1612. [PubMed: 27357569]

12. Burke KA, et al. Residue-by-residue view of In vitro FUS granules that bind the C-terminal domain of RNA polymerase II. Mol Cell. 2015; 60:231-241. [PubMed: 26455390]

13. Elbaum-Garfinkle $S$, et al. The disordered $P$ granule protein LAF-1 drives phase separation into droplets with tunable viscosity and dynamics. Proc Natl Acad Sci U S A. 2015; 112:7189-7194. [PubMed: 26015579]

14. Lin Y, et al. Formation and maturation of phase-separated liquid droplets by RNA-binding proteins. Mol Cell. 2015; 60:208-219. [PubMed: 26412307]

15. Molliex A, et al. Phase separation by low complexity domains promotes stress granule assembly and drives pathological fibrillization. Cell. 2015; 163:123-133. [PubMed: 26406374]

16. Murakami T, et al. ALS/FTD mutation-induced phase transition of FUS liquid proplets and reversible hydrogels into irreversible hydrogels impairs RNP granule function. Neuron. 2015; 88:678-690. [PubMed: 26526393]

17. Nott TJ, et al. Phase transition of a disordered nuage protein generates environmentally responsive membraneless organelles. Mol Cell. 2015; 57:936-947. [PubMed: 25747659]

18. Xiang S, et al. The LC domain of hnRNPA2 adopts similar conformations in hydrogel polymers, liquid-like droplets, and nuclei. Cell. 2015; 163:829-839. [PubMed: 26544936]

19. Zhang H, et al. RNA controls PolyQ protein phase transitions. Mol Cell. 2015; 60:220-230. [PubMed: 26474065]

20. Pak CW, et al. Sequence determinants of intracellular phase separation by complex coacervation of a disordered protein. Mol Cell. 2016; 63:72-85. [PubMed: 27392146]

21. Smith J, et al. Spatial patterning of $P$ granules by RNA-induced phase separation of the intrinsically-disordered protein MEG-3. eLife. 2016; 5:e21337. [PubMed: 27914198]

22. Conicella AE, et al. ALS mutations disrupt phase separation mediated by a-helical structure in the TDP-43 low-complexity C-terminal domain. Structure. 2016; 24:1537-1549. [PubMed: 27545621]

23. Ryan VH, et al. Mechanistic view of hnRNPA2 low-complexity domain structure, interactions, and phase separation altered by mutation and arginine methylation. Mol Cell. 2018

24. Mitrea DM, et al. Nucleophosmin integrates within the nucleolus via multi-modal interactions with proteins displaying R-rich linear motifs and rRNA. eLife. 2016; 5:e13571. [PubMed: 26836305] 
25. Uversky VN. Intrinsically disordered proteins in overcrowded milieu: Membrane-less organelles, phase separation, and intrinsic disorder. Curr Opin Struct Biol. 2016; 44:18-30. [PubMed: 27838525]

26. Zhou HX, Pang X. Electrostatic interactions in protein structure, folding, binding, and condensation. Chem Rev. 2018; 118:1691-1741. [PubMed: 29319301]

27. Flory PJ. Thermodynamics of high polymer solutions. J Chem Phys. 1942; 10:51-61.

28. Zhou HX. Polymer models of protein stability, folding, and interactions. Biochemistry. 2004; 43:2141-2154. [PubMed: 14979710]

29. Wei MT, et al. Phase behaviour of disordered proteins underlying low density and high permeability of liquid organelles. Nat Chem. 2017; 9:1118-1125. [PubMed: 29064502]

30. Chang LW, et al. Sequence and entropy-based control of complex coacervates. Nat Commun. 2017; 8:1273. [PubMed: 29097695]

31. Lin YH, Chan HS. Phase separation and single-chain compactness of charged disordered proteins are strongly correlated. Biophys J. 2017; 112:2043-2046. [PubMed: 28483149]

32. Saha S, et al. Polar positioning of phase-separated liquid compartments in cells regulated by an mRNA competition mechanism. Cell. 2016; 166:1572-1584 e1516. [PubMed: 27594427]

33. Banerjee PR, et al. Reentrant phase transition drives dynamic substructure formation in ribonucleoprotein droplets. Angew Chem Int Ed Engl. 2017; 56:11354-11359. [PubMed: 28556382]

34. Nguemaha V, Zhou HX. Liquid-liquid phase separation of patchy particles illustrates disparate effects of constituents on protein droplets. Sci Rep. 2018 submitted.

35. Taratuta VG, et al. Liquid-liquid phase separation of aqueous lysozyme solutions: effects of $\mathrm{pH}$ and salt identity. J Phys Chem. 1990; 94:2140-2144.

36. Broide ML, et al. Binary-liquid phase separation of lens protein solutions. Proc Natl Acad Sci U S A. 1991; 88:5660-5664. [PubMed: 2062844]

37. Tombs MP, et al. Protein solubility: Phase separation in arachin-salt-water systems. Int J Peptide Protein Res. 1974; 6:253-277. [PubMed: 4426737]

38. Tanaka T, et al. Phase separation of a protein-water mixture in cold cataract in the young rat lens. Science. 1977; 197:1010-1012. [PubMed: 887936]

39. ten Wolde PR, Frenkel D. Enhancement of protein crystal nucleation by critical density fluctuations. Science. 1997; 277:1975-1978. [PubMed: 9302288]

40. Gliko O, et al. A metastable prerequisite for the growth of lumazine synthase crystals. J Am Chem Soc. 2005; 127:3433-3438. [PubMed: 15755162]

41. Veesler S, et al. Crystallization in the presence of a liquid- liquid phase separation. Org Process Res Dev. 2006; 10:841-845.

42. Schubert R, et al. Real-time observation of protein dense liquid cluster evolution during nucleation in protein crystallization. Cryst Growth Des. 2017; 17:954-958.

43. Annunziata $\mathrm{O}$, et al. Observation of liquid-liquid phase separation for eye lens gammaS-crystallin. Proc Natl Acad Sci U S A. 2003; 100:970-974. [PubMed: 12529503]

44. Wang Y, et al. Phase separation in solutions of monoclonal antibodies and the effect of human serum albumin. Proc Natl Acad Sci U S A. 2011; 108:16606-16611. [PubMed: 21921237]

45. Wang Y, et al. Liquid-liquid phase separation in oligomeric peptide solutions. Langmuir. 2017; 33:7715-7721. [PubMed: 28689408]

46. Qin S, Zhou HX. Fast method for computing chemical potentials and liquid-liquid phase equilibria of macromolecular solutions. J Phys Chem B. 2016; 120:8164-8174. [PubMed: 27327881]

47. Patel A, et al. A liquid-to-solid phase transition of the ALS protein FUS accelerated by disease mutation. Cell. 2015; 162:1066-1077. [PubMed: 26317470]

48. Protter DSW, et al. Intrinsically disordered regions can contribute promiscuous interactions to RNP granule assembly. Cell Rep. 2018; 22:1401-1412. [PubMed: 29425497]

49. Banjade $S$, et al. Conserved interdomain linker promotes phase separation of the multivalent adaptor protein Nck. Proc Natl Acad Sci U S A. 2015; 112:E6426-6435. [PubMed: 26553976]

50. Harmon TS, et al. Intrinsically disordered linkers determine the interplay between phase separation and gelation in multivalent proteins. Elife. 2017; 6 
51. Rabouille C, Alberti S. Cell adaptation upon stress: the emerging role of membrane-less compartments. Curr Opin Cell Biol. 2017; 47:34-42. [PubMed: 28342303]

52. Joyner RP, et al. A glucose-starvation response regulates the diffusion of macromolecules. eLife. 2016; 5:e09376. [PubMed: 27003290]

53. Monahan Z, et al. Phosphorylation of the FUS low-complexity domain disrupts phase separation, aggregation, and toxicity. EMBO J. 2017; 36:2951-2967. [PubMed: 28790177]

54. Wang JT, et al. Regulation of RNA granule dynamics by phosphorylation of serine-rich, intrinsically disordered proteins in C. elegans. eLife. 2014; 3:e04591. [PubMed: 25535836]

55. Kmoch S, et al. Link between a novel human gamma D-crystallin allele and a unique cataract phenotype explained by protein crystallography. Hum Mol Genet. 2000; 9:1779-1786. [PubMed: 10915766]

56. Pande A, et al. Crystal cataracts: human genetic cataract caused by protein crystallization. Proc Natl Acad Sci U S A. 2001; 98:6116-6120. [PubMed: 11371638]

57. San Biagio PL, Palma MU. Spinodal lines and Flory-Huggins free-energies for solutions of human hemoglobins HbS and HbA. Biophys J. 1991; 60:508-512. [PubMed: 1912284]

58. Galkin $\mathrm{O}$, et al. Liquid-liquid separation in solutions of normal and sickle cell hemoglobin. Proc Natl Acad Sci U S A. 2002; 99:8479-8483. [PubMed: 12070342]

59. Munder MC, et al. A pH-driven transition of the cytoplasm from a fluid- to a solid-like state promotes entry into dormancy. eLife. 2016; 5:e09347. [PubMed: 27003292]

60. Mackenzie IR, et al. TIA1 mutations in amyotrophic lateral sclerosis and frontotemporal dementia promote phase separation and alter stress granule dynamics. Neuron. 2017; 95:808-816. [PubMed: 28817800]

61. Lee TD, Yang CN. Statistical theory of equations of state and phase transitions. II. Lattice gas and Ising model. Phys Rev. 1952; 87:410-419.

62. Muthukumar M. Thermodynamics of polymer solutions. J Chem Phys. 1986; 85:4722-4728.

63. Quiroz FG, Chilkoti A. Sequence heuristics to encode phase behaviour in intrinsically disordered protein polymers. Nat Mater. 2015; 14:1164-1171. [PubMed: 26390327]

64. Simon JR, et al. Programming molecular self-assembly of intrinsically disordered proteins containing sequences of low complexity. Nat Chem. 2017; 9:509-515. [PubMed: 28537592]

65. Ambadipudi S, et al. Liquid-liquid phase separation of the microtubule-binding repeats of the Alzheimer-related protein Tau. Nat Commun. 2017; 8:275. [PubMed: 28819146]

66. Riback JA, et al. Stress-triggered phase separation is an adaptive, evolutionarily tuned response. Cell. 2017; 168:1028-1040. e1019. [PubMed: 28283059]

67. Carnahan NF, Starling KE. Equation of state for nonattracting rigid spheres. J Chem Phys. 1969; 51:635-636.

68. Barker JA, Henderso D. Perturbation theory and equation of state for fluids: The square-well potential. J Chem Phys. 1967; 47:2856-2861.

69. Chang J, Sandler SI. A real function representation for the structure of the hard-sphere fluid. Mol Phys. 1994; 81:735-744.

70. Vliegenthart GA, Lekkerkerker HNW. Predicting the gas-liquid critical point from the second virial coefficient. J Chem Phys. 2000; 112:5364-5369.

71. Velasco E, et al. Phase diagram of colloidal systems. Langmuir. 1998; 14:5652-5655.

72. Gast AP, et al. Polymer-induced phase separations in non-aqueous colloidal suspensions. J Colloid Interf Sci. 1983; 96:251-267.

73. Choi Y, et al. Hard-sphere radial distribution functions for face-centered cubic and hexagonal close-packed phases: Representation and use in a solid-state perturbation theory. J Chem Phys. 1991; 95:7548-7561.

74. Wertheim MS. Thermodynamic perturbation-theory of polymerization. J Chem Phys. 1987; 87:7323-7331.

75. Escobedo FA, De Pablo JJ. Chemical-potential and equations of state of hard-core chain molecules. J Chem Phys. 1995; 103:1946-1956.

76. Escobedo FA, De Pablo JJ. Simulation and prediction of vapour-liquid equilibria for chain molecules. Mol Phys. 1996; 87:347-366. 
77. Peng K, et al. Length-dependent prediction of protein intrinsic disorder. BMC Bioinformatics. 2006; 7:208. [PubMed: 16618368]

78. Berland CR, et al. Solid-liquid phase boundaries of lens protein solutions. Proc Natl Acad Sci U S A. 1992; 89:1214-1218. [PubMed: 1741375]

\section{Glossary}

Coexistence curve

Critical point

IDR

IDP

LLPS

MLO

PTM

RRM a relation between two thermodynamic properties, such as temperature and pressure or temperature and concentration, that is followed when two phases are at equilibrium. Also known as phase boundary.

a unique thermodynamic condition at which distinction between two fluid phases (gas and liquid for simple molecular fluids or dispersed and droplet for macromolecular solutions) vanishes. In particular, the concentrations of the two phases become identical and are known as the critical concentration. Phase separation occurs only on one side of the critical point, e.g., below the critical temperature.

intrinsically disordered region

intrinsically disordered protein

liquid-liquid phase separation

membraneless organelle

posttranslational modification

RNA-recognition motif, an amino-acid sequence motif that consists of approximately 90 residues and forms a structural fold with a four-stranded $\beta$-sheet packed against two a-helices 


\section{Box 1}

\section{Phases and determination of coexistence curves}

For simple molecules like water, the separation between two phases occurs when the molecules can achieve the same low free energy by adopting two distinct types of configurations, with different densities and different extents of intermolecular contacts (Figure Ia). The low free energy may result from high entropy in one phase (e.g., with high translational freedom for the molecules) and strong negative enthalpy in the other phase (e.g., with favorable intermolecular interactions). A homogeneous mix of the two phases, with a density falling in the gap between the two phases, would have a higher free energy and hence only be metastable relative to the coexistence of the two phases. The three common phases are gas, liquid, and solid (Figure Ib).

For two phases to coexist, they have to satisfy thermodynamic conditions, namely, equality of temperature $T$, pressure $P$, and chemical potential $\mu$, since the two phases are in contact and are equally stable. The chemical potential itself is a function of

$T$ and $P$, so the equality in $\mu$ between the phases defines a relation between $T$ and $P$, i.e., a coexistence curve in the $T-P$ plane that separates the two phases (Figure Ib). On one side of the coexistence curve, one phase has a lower chemical potential and hence is the stable phase; the opposite is true on the other side of the coexistence curve. Phase boundaries can continue indefinitely, or terminate at a certain point. In particular, the boundary between the gas and liquid phases starts at the "triple" point (where gas, liquid, and solid coexist) and ends at the critical point (where the distinction between gas and liquid vanishes). The density $\rho$ is also a function of $T$ and $P$, but typically experiences a discontinuity across a phase boundary. So on the $\rho-T$ plane, a coexistence curve between two phases has two branches; the regions outside the two branches correspond to two stable phases whereas the region within the two branches is only metastable (Figure Ic).

The determination of the phase boundary requires the calculation of the free energy as a function of density for a given temperature. In the double-tangent construction, one plots $f \equiv F / V$, the Helmholtz free energy per unit volume, as a function of density. Phase separation occurs when $f$ is tangent to a single line at two points (Figure Ia). The two points of tangency identify the respective densities of the two coexisting phases; the slope of the line gives the chemical potential (because $\mu=\partial f / \partial \rho$ ); and the negative of the intercept with the $f$ axis yields the pressure (because $f=\mu \rho-P$ ). Equivalently, the densities of the two coexisting phases can be identified by the Maxwell construction in the $\mu-\rho$ plane, involving a horizontal line that intersects the isotherm loop with equal areas above and below.

The principle outlined above applies equally to protein-solvent mixtures. The role of the solvent can be treated as modifying the interactions between protein molecules; the pressure relevant for phase equilibria is the osmotic pressure due to the protein rather than the total pressure of the protein mixture, and vanishes at zero protein concentration (as would be true also for an ideal gas at zero density). Accordingly proteins can also exist in three phases. The typical aqueous solutions of proteins correspond to the liquid 
phase; solutions of structured proteins at high concentrations can crystalize, creating the solid phase; lastly, solutions of disordered proteins often can easily separate into two phases, with protein-rich droplets coexisting with a protein-poor solution. This LLPS isreminiscent of liquid-gas coexistence for simple molecular fluids. Light scattering by the protein droplets (typically microns in size) gives the protein solution a cloudy appearance (in the same way as our eyes see clouds in the sky). 


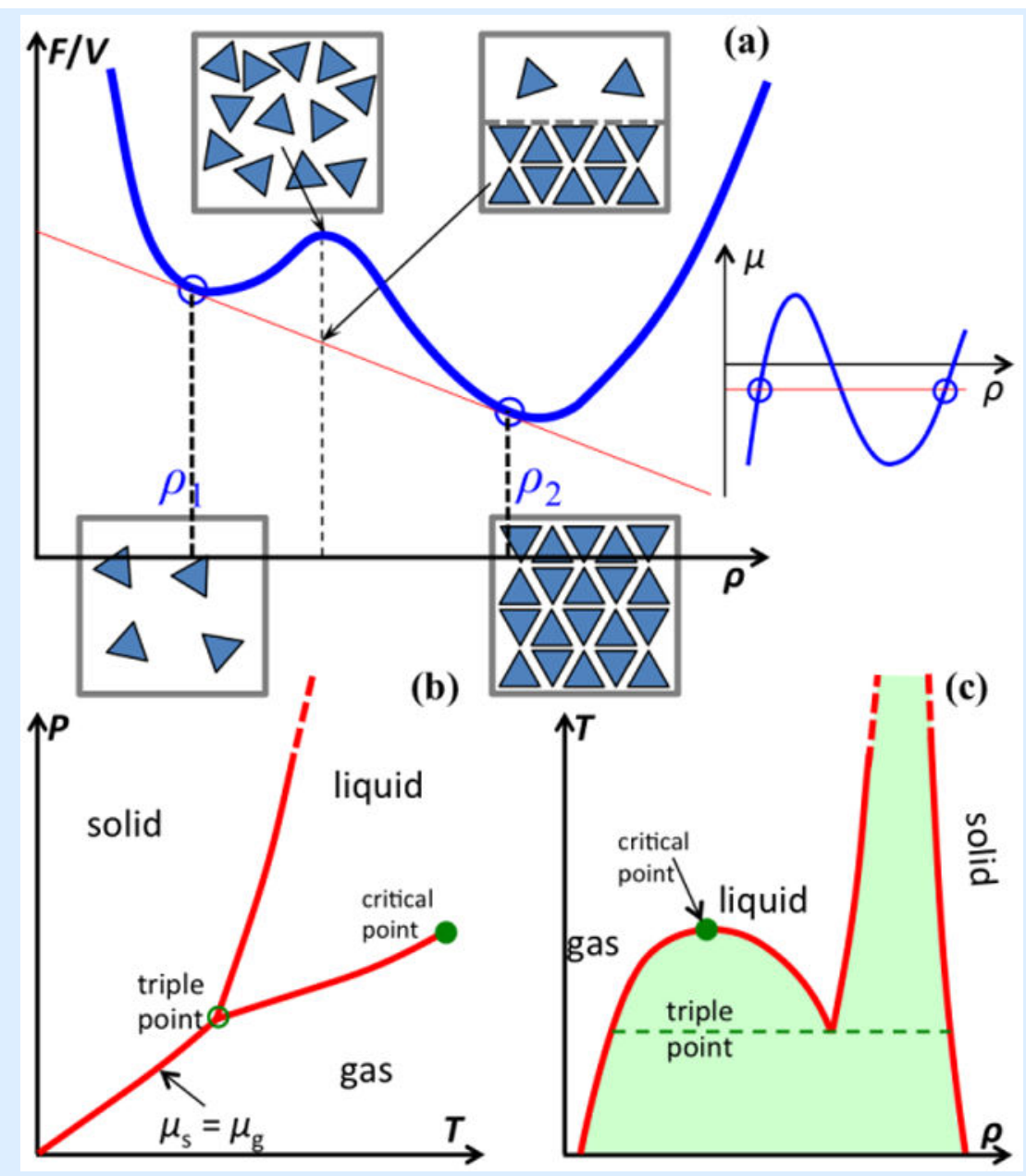

Figure I.

Phase boundaries. (a) Free energy per unit volume, $f \equiv F / V$, as a function of density, $\rho$. Two phases emerge when this function (blue curve) is tangent to a single line (in red) at two points (indicated by circles). The two bottom boxes illustrate molecular configurations in two stable phases; the top left box represents a metastable configuration, whereas the top right box represents the separation of two phases. On the far right is an illustration of the Maxwell construction in the $\mu-\rho$ plane, where the red line dissects the isotherm (blue curve) with equal areas. (b) Boundaries, on the $T-P$ plane, between three common phases. (c) Coexistence curves on the $\rho-T$ plane. Metastable regions between phases are shaded. 
Box 2

\section{Free energy calculation: lattice models and mean-field treatment}

Lattice models, where particles occupy sites on a defined lattice, have been very useful in illustrating basic physical ideas by simplifying free energy calculations [61]. For example, consider a lattice with each site occupied by one and only one molecule, of which there are two types, A and S, representing solute and solvent, respectively (Figure Ia top). Suppose that the numbers of $\mathrm{A}$ and $\mathrm{S}$ molecules are $M_{\mathrm{A}}$ and $M_{\mathrm{S}}$, respectively, and the volume of each lattice site is $v_{1}$. Then the volumes occupied by $\mathrm{A}$ and $\mathrm{S}$ molecule are $V_{A}=M_{\mathrm{A}} v_{1}$ and $V_{\mathrm{S}}=M_{\mathrm{S}} v_{1}$, respectively, and the total number of sites is $M=M_{\mathrm{A}}+M_{\mathrm{S}}$ and the total volume of the lattice is $V=V_{\mathrm{A}}+V_{\mathrm{S}}=M v_{1}$. Relative to the unmixed case (where the $\mathrm{A}$ and $\mathrm{S}$ molecules each occupy their own lattices with respective volumes $V_{\mathrm{A}}$ and $V_{\mathrm{S}}$ ), the lattice of a totally random A-S mixture has an increase, $\Delta S_{\mathrm{mix}}$, in entropy,

$$
\Delta \mathrm{S}_{\text {mix }}=M_{\mathrm{A}} k_{\mathrm{B}} \ln \left(V / V_{\mathrm{A}}\right)+M_{\mathrm{S}} k_{\mathrm{B}} \ln \left(V / V_{S}\right) \quad[\mathrm{I}]
$$

similar to what happens upon the expansion of an ideal gas. Here $k_{\mathrm{B}}$ denotes the Boltzmann constant. In terms of the volume fraction $\phi=V_{\mathrm{A}} / V$ of the solute, we have

$$
\frac{\Delta S_{\text {mix }}}{M k_{\mathrm{B}}}=-\phi \ln \phi-(1-\phi) \ln (1-\phi)
$$

Note that the rate of entropy change with respect to $\phi$ is the greatest as $\phi \rightarrow 0$ or 1 .

To obtain the free energy, we still need the average energy of the A-S mixture. To that end, we assume that each molecule has $z$ neighbors, and the interaction with each neighbor makes an energetic contribution of $\varepsilon_{\mathrm{AA}}, \varepsilon_{\mathrm{AS}}$, or $\varepsilon_{\mathrm{SS}}$, depending on the identities of the molecules in the pair. This "mean-field" treatment leads to the following average energy for the mixture:

$$
U=\frac{1}{2} M_{\mathrm{A}} z\left[\phi \varepsilon_{\mathrm{AA}}+(1-\phi) \varepsilon_{\mathrm{AS}}\right]+\frac{1}{2} M_{\mathrm{S}} z\left[\phi \varepsilon_{\mathrm{AS}}+(1-\phi) \varepsilon_{\mathrm{SS}}\right]
$$

The change in energy relative to the unmixed case is

$$
\beta \Delta U / M=\chi \phi(1-\phi) \quad[\mathrm{IV}]
$$

where $\beta=1 / k_{\mathrm{B}} T$ and 


$$
\chi=\frac{1}{2} \beta\left(\varepsilon_{\mathrm{AA}}+\varepsilon_{\mathrm{SS}}-2 \varepsilon_{\mathrm{AS}}\right) z
$$

which measures the magnitude of the effective attraction between two A molecules. Combining Equations [II] and [IV], we find the Helmholtz free energy as

$$
v_{1} \beta f=\phi \ln \phi+(1-\phi) \ln (1-\phi)+\chi \phi(1-\phi)+\phi v_{1} \beta f_{\mathrm{A}}+(1-\phi) v_{1} \beta f_{\mathrm{S}}
$$

where the second line represents the free energy of the unmixed case. The A-S mixture can separate into an A-poor phase and an A-rich phase (Figure Ib). The critical point, where the two branches of the coexistence curve coalesce, can be found by requiring both the second and third derivatives of $f$ with respect to $\phi$ to vanish, yielding $\phi_{\mathrm{c}}=\frac{1}{2}$ for the critical volume fraction and $\chi_{\mathrm{c}}=2$ for the critical value of $\chi$. Phase separation occurs only when $\chi \geq \chi_{\mathrm{c}}$ (i.e., A-A attraction must exceed a critical strength); here the two branches of the coexistence curve are symmetric with respect to $\phi=\phi_{\mathrm{c}}$.

Flory and Huggins [27] developed a lattice model for calculating the entropy for mixing polymer chains with solvent molecules. Each lattice site was occupied by either a monomer unit (like an A molecule) of a polymer or a solvent molecule; two adjacent monomer units along a polymer occupied neighboring sites (Figure Ia bottom). Suppose that there are $N$ polymers, each with chain length $L$. The entropy of the polymer-solvent mixture can be calculated by counting the number of ways that the polymers can be placed in the lattice. For the first polymer, the first monomer unit has $M$ choices for its site; the second unit has $z$ choices; and all subsequent units each have $z-1$ choices. However, the fact that no more than one monomer unit can occupy the same site reduces the number of choices; the reduction factors are $(M-1) / M$ for the second unit, $(M-$ $2) / M$ for the third unit, etc. Therefore the total number of ways for placing the first polymer is

$$
\begin{aligned}
v_{1}=M \frac{z(M-1)}{M} & \frac{(z-1)(M-2)}{M} \cdots \frac{(z-1)(M-L+1)}{M} \\
& \approx\left(\frac{z-1}{M}\right)^{L-1} \frac{M !}{(M-L) !}
\end{aligned}
$$

Similarly, if $n$ polymers have already been placed, occupying $n L$ sites, the total number of ways for placing the $(n+1)$ th polymer is

$$
v_{n+1}=\left(\frac{z-1}{M}\right)^{L-1} \frac{(M-n L) !}{[M-(n+1) L] !}
$$


The total number of ways for placing $N$ polymers on a lattice with $M$ sites is then

$$
\Omega(N, M)=\frac{1}{! N} \prod_{n=1}^{N} v_{n}=\frac{1}{N !}\left(\frac{z-1}{M}\right)^{N(L-1)} \frac{M !}{(M-N L) !}
$$

where a factor $1 / N$ ! has been inserted to account for the indistinguishability of the polymers. Finally the mixing entropy is

$$
\frac{\Delta S_{\operatorname{mix}}}{k_{\mathrm{B}}}=\ln \frac{\Omega(N, M)}{\Omega(N, N l) \Omega(0, M-N L)}
$$

or

$$
\frac{\Delta S_{\mathrm{mix}}}{M k_{\mathrm{B}}}=-\frac{\phi}{L} \ln \phi-(1-\phi) \ln (1-\phi)
$$

where $\phi=N L / M$ is the volume fraction of the polymers. Notice that the last expression correctly reduces to Equation [II] when $L=1$. At a high degree of polymerization (i.e., $L$ $\gg 1$ ), the rate of entropy change with respect to $\phi$ near $\phi=0$ is significantly less than that for the A-S mixture, because the polymers, due to chain connectivity between monomer units, are significantly less mixed with the solvent. Combined with the mean-field treatment of the average energy (see Equation [VI]), the free energy of the polymersolvent mixture is

$$
v_{1} \beta f=\frac{\phi}{L} \ln \phi+(1-\phi) \ln (1-\phi)+\chi \phi(1-\phi)+\phi v_{1} \beta f_{\mathrm{p}}+(1-\phi) v_{1} \beta f_{\mathrm{s}}
$$

where $\chi$ now represents the magnitude of the monomer-monomer attraction, and the second line represents the free energy of the unmixed case. Effects beyond the mean-field treatment, including monomer density fluctuation and three-body or sequence-dependent interaction, have also been treated theoretically [31, 62].

For the polymer solution, the coexistence curve between the polymer-poor and polymerrich phases becomes asymmetric (Figure Ib), due to the reduced mixing entropy at $\phi$ near 0 (relative to the counterpart at $\phi$ near 1). The critical point is at $\phi_{\mathrm{c}}=1 /(1+\sqrt{ } L)$ and $\chi_{\mathrm{c}}=\frac{1}{2}(1+1 / \sqrt{L})^{2}$. As the polymer chain length increases from 1 to $\infty$, the critical volume fraction decreases from 0.5 to 0 while $\chi_{\mathrm{c}}$ decreases from 2 to 0.5 (the latter change corresponding to a 4 -fold increase in the critical temperature $T_{\mathrm{c}}$ ). So for long polymer chains, LLPS occurs at low concentrations and over a wide range of temperatures. 
The latter is a qualitatively important result for polymer solutions. However, the lattice model underlying the Flory-Huggins theory provides only a very crude account of excluded-volume effects (in particular, resulting in unphysically high $\phi$ values in the polymer-rich phase shown in Figure Ib). Moreover, the mean-field treatment yields only a rough estimate for the effects of solute-solute attraction. Box 3 and Box 4 present much more realistic calculations of these effects for colloids and polymers, respectively.

The Flory-Huggins theory predicts only upper critical solution temperature (UCST), a situation where $T_{\mathrm{c}}$ is the upper bound of temperatures for phase separation. Polymers are also known to have lower critical solution temperature (LCST), in which case $T_{\mathrm{c}}$ is the lower bound of temperatures where LLPS occurs. By making $k_{\mathrm{B}} T_{\chi}$ dependent on $T$ and/or $\phi$, LCST can be predicted. LCST has been observed on synthetic peptides and on some proteins [63-66]; better physical understanding of such observations will be desirable. 
(a)
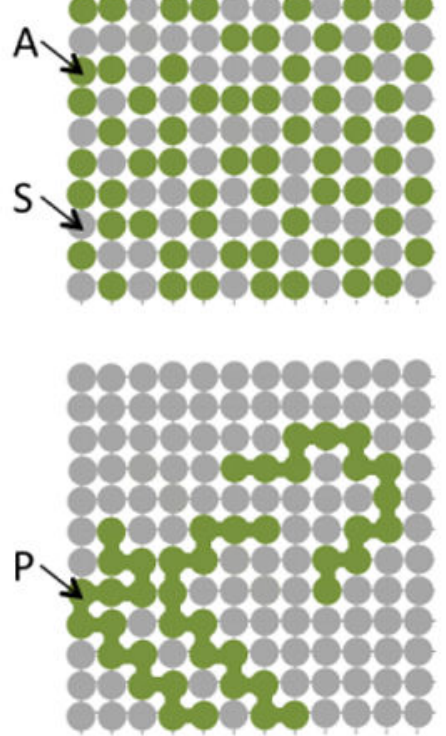

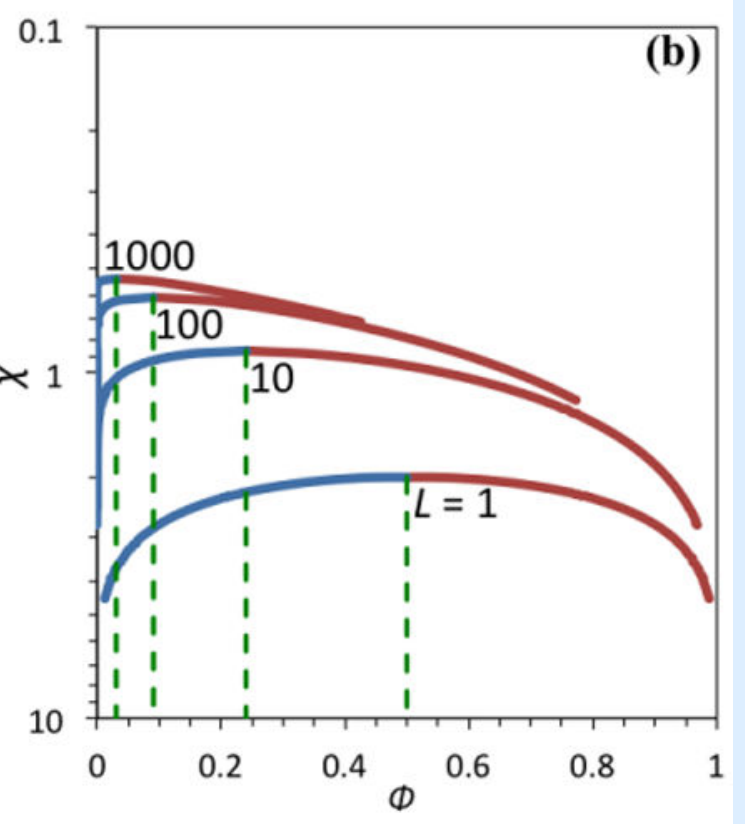

(b)

Figure I.

Lattice models and their liquid-liquid phase separation. (a) Lattice models for a mixture of A molecules (top) or polymers (bottom) with S molecules. Each A or S molecule as well as each monomer of the polymers occupies one and only one site on the lattice. (b) Coexistence curves for polymers chains of various lengths. The polymer-poor and polymer-rich branches are in blue and cayenne, respectively. The $L=1$ case is equivalent to the A-S mixture. 
Box 3

\section{Free energy calculations: perturbation theory for colloids}

Both simple molecular fluids and colloidal suspensions have often been modeled by an inter-particle interaction comprising a hard-core repulsion and a short-range attraction. The square-well potential is an example of such an interaction:

$$
u(r)=\left\{\begin{array}{l}
\infty, \quad r<\sigma \\
-\varepsilon, \quad \sigma<x<\sigma+\delta \quad[\mathrm{I}] \\
0, \quad r>\sigma+\delta
\end{array}\right.
$$

where $r$ is the inter-particle distance, $\sigma$ is the diameter of hard core, and $\delta$ and $\varepsilon$ are the width and depth, respectively, of the attraction. Without the attraction, the particles are hard spheres, for which an accurate expression for the free energy per particle is [67]

$$
\frac{\beta F_{\mathrm{HS}}}{N}=\ln \left(\rho v_{Q}\right)-1+\frac{\phi(4-3 \phi)}{(1-\phi)^{2}}
$$

where $v_{\mathrm{Q}}$ is an arbitrary constant with the dimension of volume, and $\phi=\rho v_{\mathrm{p}}$, with $\nu_{\mathrm{p}}=$ $\pi \sigma^{3} / 6$ denoting the volume of each particle, is the volume fraction of the particles. The first two terms of Equation [II] constitute the "ideal" part of the free energy whereas the last term is the excess part due to hard-core repulsion. The effect of the attraction can be obtained by a perturbation theory [68]. To the first order, the theory predicts

$$
\frac{\beta F}{N}=\frac{\beta F_{\mathrm{HS}}}{N}-\frac{1}{2} \beta \varepsilon \rho \int_{\rho}^{\sigma+\rho} g_{\mathrm{HS}}(r) 4 \pi r^{2} d r
$$

where $g_{\mathrm{HS}}(r)$ is the radial distribution function, representing the relative density of other hard spheres around a tagged hard sphere. On a per unit volume basis, we have [35]

$$
v_{\mathrm{p}} \beta f=\phi \ln \left(\frac{\phi v_{\mathrm{Q}}}{v_{\mathrm{p}}}\right)-\phi+\frac{\phi^{2}(4-3 \phi)}{(1-\phi)^{2}}-\chi \phi^{2}
$$

The parameter

$$
\chi=12 \beta \varepsilon \int_{1}^{1+\delta / \sigma} g_{\mathrm{HS}}(x \sigma) x^{2} d x \equiv 12 \beta \varepsilon I(\phi ; \delta / \sigma)
$$


as in Box I, measures the strength of inter-particle attraction. The integral $I(\phi ; \delta / \sigma)$ [69] can be fitted to a quadratic function $b_{0}+b_{1} \phi+b_{2} \phi^{2}$, and the coefficients $b_{0}, b_{1}$, and $b_{2}$ are $0.178,0.258$, and 1.01 , respectively, at $\delta / \sigma=0.15$ for $\phi<0.49$. The corresponding expression for the chemical potential is

$$
\beta \mu=\ln \left(\frac{\phi v_{\mathrm{Q}}}{v_{\mathrm{p}}}\right)+\frac{\phi\left(8-9 \phi+9 \phi^{2}\right)}{(1-\phi)^{3}}-24 \beta \varepsilon \phi\left(b_{0}+3 b_{1} \phi / 2+2 b_{2} \phi^{2}\right)
$$

Equation [IV] leads to LLPS (Figure I). The critical point would be at $\phi_{\mathrm{c}}=0.13$ and $\chi_{\mathrm{c}}=$ 10.6 if $I(\phi ; \delta / \sigma)$ (and hence $\chi$ ) were independent of $\phi$ (as would be in a mean-field treatment) [35]. Note that a $\phi$-independent $-\chi$ can be viewed as the lowest order coefficient, $\left(B_{2}-B_{2 ; \mathrm{HS}}\right) / \nu_{\mathrm{p}}$, of a "perturbative" virial expansion of $\nu_{\mathrm{p}} \beta f$, where $B_{2}$ is the second virial coefficient and $B_{2 ; \mathrm{HS}}=4 \nu_{\mathrm{p}}$ is the counterpart for the hard-sphere reference. The preceding $\chi_{\mathrm{c}}$ value corresponds to $-B_{2}\left(T_{\mathrm{c}}\right) / \nu_{\mathrm{p}}=6.6$; a near constancy of $-B_{2}\left(T_{\mathrm{c}}\right) / \nu_{\mathrm{p}}$ around 6 was first proposed by Vliegenthart and Lekkerkerker [70]. It is apparent from Equation $[\mathrm{V}]$ that the critical temperature increases with increasing magnitude and range of the attraction. For example, as $\delta / \sigma$ increases from 0.05 to $0.15, k_{\mathrm{B}} T_{\mathrm{c}} / \mathcal{\varepsilon}$ increases from 0.286 to 0.670 when the $\phi$ dependence of $\chi$ is accounted for. Hard-sphere liquids, corresponding to $\chi=0$, do not have LLPS.

Hard spheres do undergo a fluid-solid phase transition, forming crystals with a facecentered cubic (FCC) unit cell. The free energy in the solid phase can be estimated from a free-volume approach [71] as

$$
\frac{\beta F_{\mathrm{HS}}^{\mathrm{S}}}{N}=\ln \left(\frac{v_{\mathrm{Q}}}{v_{\mathrm{F}}}\right)
$$

where a subscript "s" indicates the solid phase, and

$$
v_{\mathrm{F}}=\frac{8}{\sqrt{2}}\left(R_{1}-\sigma\right)^{3} \quad[\mathrm{VIII}]
$$

is the volume accessible to a hard sphere in the FCC lattice. In the last expression, $R_{1}$ denotes the distance between nearest neighbors, which is related to the density of the crystal via $1 / \rho=\frac{1}{\sqrt{2}} R_{1}^{3}$. With Equations [II] and [IV] for the free energies of the fluid and solid, the coexistence volume fractions are found to be 0.49 and 0.56 , respectively, for the two phases.

The effect of the inter-particle attraction in the solid phase can also be obtained by a perturbation theory, similar to Equation [III] [72]. After fitting the integral of the radial distribution function of the hard-sphere FCC solid [73] to a quadratic function of $\phi$ (cf. Equation $[\mathrm{V}])$, the free energy is 


$$
\frac{\beta F^{\mathrm{S}}}{N}=\frac{\beta F_{\mathrm{HS}}^{\mathrm{S}}}{N}-12 \beta \varepsilon \phi\left[c_{0}-c_{1}\left(\phi-\phi_{0}\right)^{2}\right] \quad[\mathrm{IX}]
$$

where the parameters $c_{0}, c_{1}$, and $\phi_{0}$ are $0.767,12.8$, and 0.643 , respectively, at $\delta / \sigma=0.15$ for $0.52 \leq \phi \leq 0.73$. Using Equations [III] and [IX], the coexistence curve between the fluid and solid phases can be determined (Figure I). Note that the coexistence curve for LLPS falls entirely in the metastable region for fluid-solid phase separation. Therefore for colloids and structured proteins that can form crystals, the thermodynamically stable coexistence is between a dissolved phase and a crystalline phase, not between two liquid phases. Whereas the ranges of attraction are comparable for macromolecular particles and simple molecules, the much smaller hard-core diameters of the latter increase the order of magnitude of the ratio $\delta / \sigma$ from 0.1 to 1 . For such higher $\delta / \sigma$, the coexistence curve for LLPS emerges above the fluid branch of the fluid-solid coexistence curve [39, 72], and hence simple molecules have three stable phases (i.e., gas, liquid, and solid). 


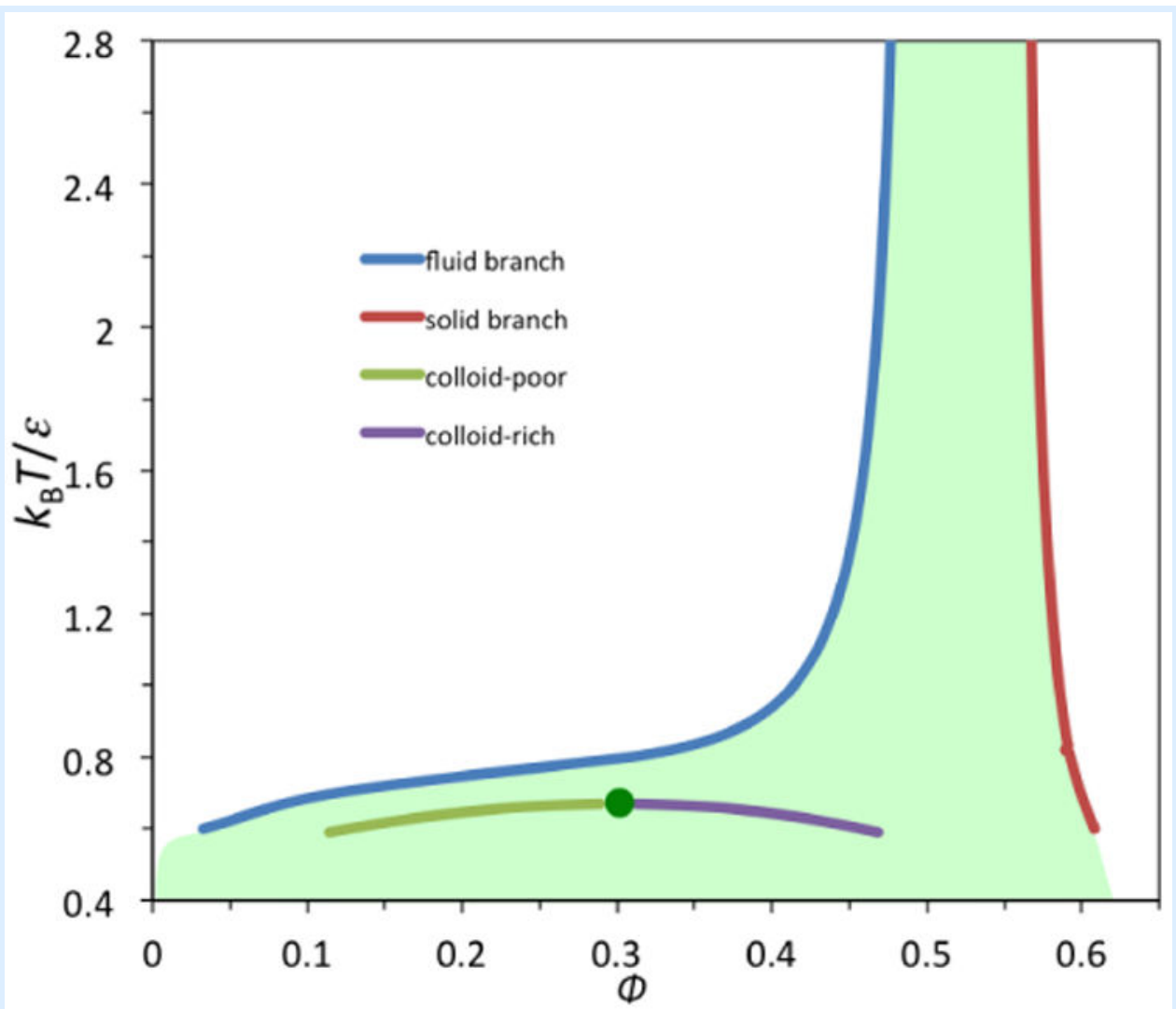

Figure I.

Coexistence curves for colloid particles modeled by a square-well potential. Note that the coexistence curve for liquid-liquid phase separation (LLPS) falls inside the metastable region (shaded) for fluid-solid phase separation. The critical point for LLPS is indicated by a green circle. 
Box 4

\section{Free energy calculations: perturbation theory for polymers}

A different type of perturbation theory has been developed for polymer solutions, with unchained monomers as reference and their bonding as perturbation [74]. This idea has been improved, with short blocks as reference and inter-block bonding as perturbation $[75,76]$ :

$$
\mu^{\mathrm{ex}}(L)=\left(L / l_{\mathrm{r}}\right) \mu^{\mathrm{ex}}\left(l_{\mathrm{r}}\right)+\left(L / l_{\mathrm{r}}-1\right) \mu^{\mathrm{bond}}\left(l_{\mathrm{r}}\right)
$$

where $L$ (or $l_{\mathrm{r}}$ ) is the chain length of the actual polymers (or reference blocks), $\mu^{\mathrm{ex}}(I)$ represents the excess chemical potential of pure 1 -mers at the same monomer density as the $L$-mers, and $\mu^{\text {bond }}\left(l_{\mathrm{r}}\right)$ represents the free energy for forming a bond between two $l_{\mathrm{r}^{-}}$ mer blocks. A further approximation is

$$
\mu^{\text {bond }}\left(l_{\mathrm{r}}\right)=\mu^{\mathrm{ex}}\left(2 l_{\mathrm{r}}\right)-2 \mu^{\mathrm{ex}}\left(l_{\mathrm{r}}\right)
$$

since the obvious difference between one $2 l_{\mathrm{r}}$-mer and two $l_{\mathrm{r}}$-mers is a single bond. Therefore

$$
\frac{\mu^{\mathrm{ex}}(L)}{L}=\frac{v^{\mathrm{ex}}\left(l_{\mathrm{r}}\right)}{l_{\mathrm{r}}}+\left(1-l_{\mathrm{r}} / L\right) \frac{\mu^{\mathrm{ex}}\left(2 l_{\mathrm{r}}\right)-2 \mu^{\mathrm{ex}}\left(l_{\mathrm{r}}\right)}{l_{\mathrm{r}}}
$$

which has been written on a per monomer basis. Equation [III] works well whether the polymers are purely repulsive or also have an attractive component. Escobedo and de Pablo [76] recommended using $l_{\mathrm{r}}=4$.

We now specialize to polymers modeled as fully flexible chains of tangent spheres interacting via a square-well potential (Box 3 Equation [I], but with the hard-core diameter of the monomers denoted here by $d$ ). To gain deeper insight, let us decompose $\mu^{\mathrm{ex}}(L)$ into a term $\mu^{\mathrm{rep}}(L)$, due to hard-core repulsion, and a term $\mu^{\mathrm{att}}(L)$, due to attraction:

$$
\mu^{\mathrm{ex}}(L)=\mu^{\mathrm{rep}}(L)+\mu^{\mathrm{att}}(L)
$$

The repulsion term, according to Equation [III], can be written as 


$$
\frac{\mu^{\mathrm{rep}}(L)}{L}=\frac{\mu^{\mathrm{rep}}\left(l_{\mathrm{r}}\right)}{l_{\mathrm{r}}}+\left(1-l_{\mathrm{r}} / L\right) \frac{\mu^{\mathrm{rep}}\left(2 l_{r}\right)-2 \mu^{\mathrm{rep}}\left(l_{r}\right)}{l_{r}}
$$

Escobedo and de Pablo [76] fitted their Monte Carlo simulations data for $\mu^{\mathrm{rep}}(I) / I$ at $I=I_{\mathrm{r}}$ and $I=2 l_{\mathrm{r}}$ to rational functions of the volume fraction $\phi$. Note that $\mu^{\mathrm{rep}}(I)$ is the work required to insert an 1 -mer into a solution of repulsive 1 -mers, and is an increasing function of $\phi$. Because of the chain connectivity between the two $I_{\mathrm{r}^{-}}$-mer blocks in the $2 I_{\mathrm{r}^{-}}$ mer, $\mu^{\mathrm{rep}}\left(2 l_{\mathrm{r}}\right)$ is always less than $2 \mu^{\mathrm{rep}}\left(l_{\mathrm{r}}\right)$, and the deficit grows with increasing $\phi$. Consequently the rise of $\mu^{\mathrm{rep}}(L) / L$ with increasing $\phi$ becomes moderated at larger $L$.

The attraction term can be similarly written as

$$
\frac{\mu^{\mathrm{att}}(L)}{L}=\frac{\mu^{\mathrm{att}}\left(l_{\mathrm{r}}\right)}{l_{\mathrm{r}}}+\left(1-l_{\mathrm{r}} / L\right) \frac{\mu^{\mathrm{att}}\left(2 l_{\mathrm{r}}\right)-2 \mu^{\mathrm{att}}\left(l_{\mathrm{r}}\right)}{l_{\mathrm{r}}}
$$

The simulation results of Escobedo and de Pablo [76] for $\mu^{\text {att }}\left(l_{\mathrm{r}}\right) / l_{\mathrm{r}}$ and $\left[\mu^{\mathrm{att}}\left(2 l_{\mathrm{r}}\right)-\right.$ $\left.2 \mu^{\operatorname{arr}}\left(I_{\mathrm{r}}\right)\right] / I_{\mathrm{r}}$ at $\delta / d=0.5$ are, up to $\phi=0.35$, approximated well by

$$
\begin{gathered}
\frac{\mu^{\text {att }}\left(l_{\mathrm{r}}\right)}{l_{\mathrm{r}}}=-24 \varepsilon \phi\left(b_{10}+b_{10} \phi\right) \quad[\mathrm{VII}] \\
\frac{\mu^{\text {att }}\left(2 l_{\mathrm{r}}\right)-2 \mu^{\text {att }}\left(l_{\mathrm{r}}\right)}{l_{\mathrm{r}}}=24 \varepsilon \phi\left(b_{20}+b_{21} \phi+b_{22} \phi^{2}\right) \quad[\mathrm{VIII}]
\end{gathered}
$$

where the coefficients $b_{10}, b_{11}, b_{20}, b_{21}$, and $b_{22}$ are $0.450,1.70,0.225,0.205$, and -1.60, respectively. The LLPS coexistence curves for polymers with lengths at 10, 50, 100, and 200 are shown in Figure I. Compared to the prediction of the Flory-Huggins theory (Box 2 Figure I), the coexistence curves are further skewed toward $\phi=0$. More importantly, the volume fractions of polymers in the droplet phase now fall into the physically accessible range. 


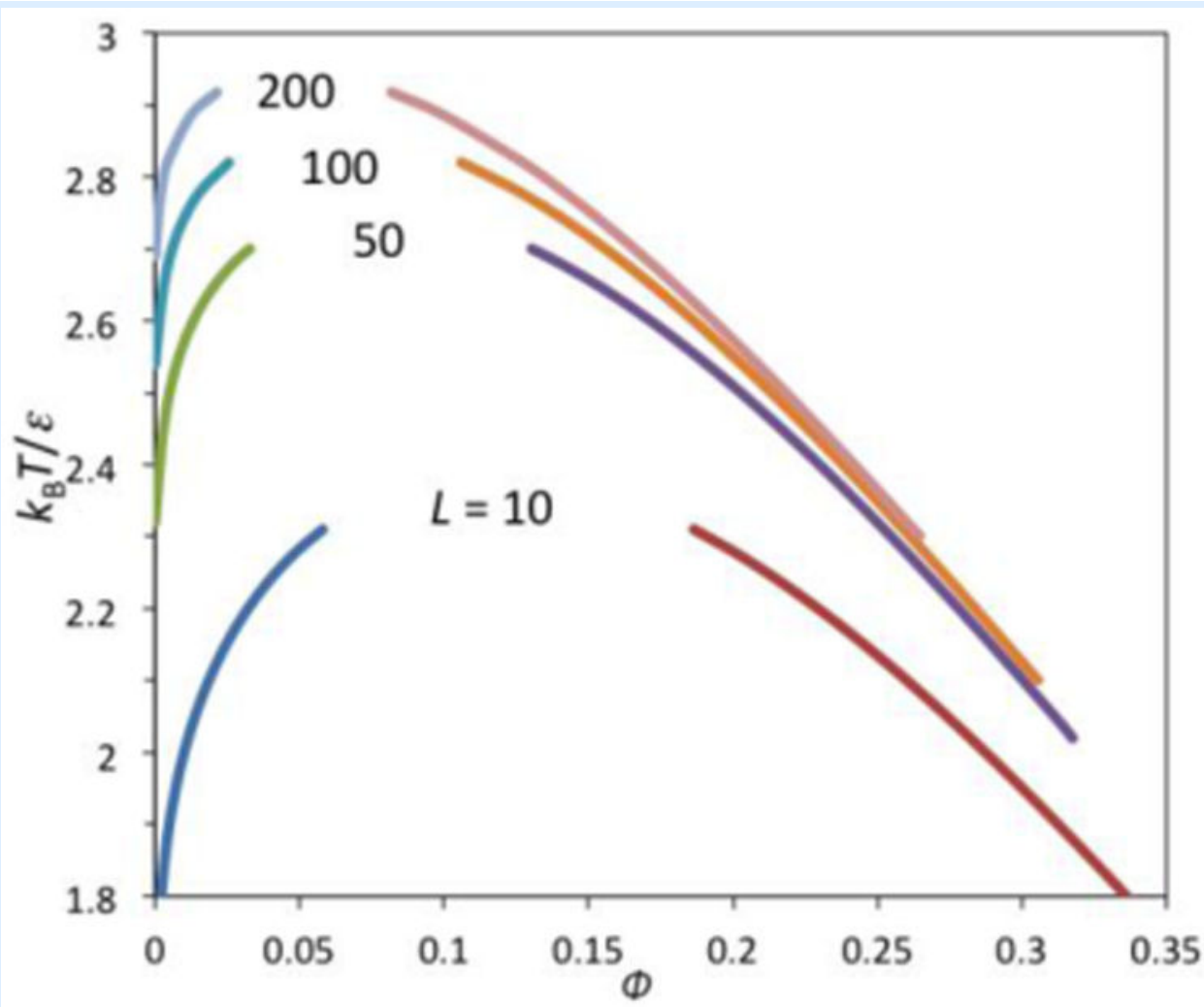

Figure I.

Coexistence curves for liquid-liquid phase separation of polymers, modeled as flexible chains of tangent square-well spheres. 


\section{Highlights}

- $\quad$ Phase separation leading to protein droplets and membraneless organelles (MLOs) is determined by the balance among translational entropy of protein molecules and steric and attractive interactions between molecules.

- Colloids and polymers serve as good models for understanding the different phase behaviors of structured and disordered proteins. Disordered proteins are characterized by both extensive attraction and low energetic cost from steric repulsion, contributing to easy observation of phase separation.

- $\quad$ Protein molecules form transient bonding networks in the droplet phase. The networks can be strengthened or disrupted by posttranslational modifications and disease-associated mutations and by other macromolecular species such as RNA, leading to assembly and disassembly of MLOs. 


\section{Outstanding Questions}

- Disordered proteins drive the formation of membraneless organelles (MLOs), but many other components help regulate the assembly/disassembly and functions of MLOs. The recruitment of certain macromolecular species into MLOs and the exclusion of many others, and the consequence on MLO assembly/disassembly are only starting to be understood in terms of intermolecular interactions. Polymer crowding agents and RNA serve as extreme examples, but the effects of the differential partitioning of many more species, their mutual influences, and their individual and collective effects on MLO structure and function are yet to be well characterized.

- Transient bonding networks appear to be a general mechanism for the internal structural organization of MLOs. Expanding the knowledge in this area is potentially a new frontier for structural biology and computational biophysics.

- Liquid-liquid coexistence curves have been determined experimentally for some proteins and more should be done. Even more urgently, calculations of coexistence curves, based on realistic modeling of proteins and rigorous implementation of thermodynamic principles, can bring tremendous physical insights to, in particular, the effects of posttranslational modifications and disease-associated mutations, but will require solutions to technical challenges.

- $\quad$ Pathogenesis of MLO proteins often involves transition to solid-like condensates. Physical models are needed to gain a foundational understanding of this process. 

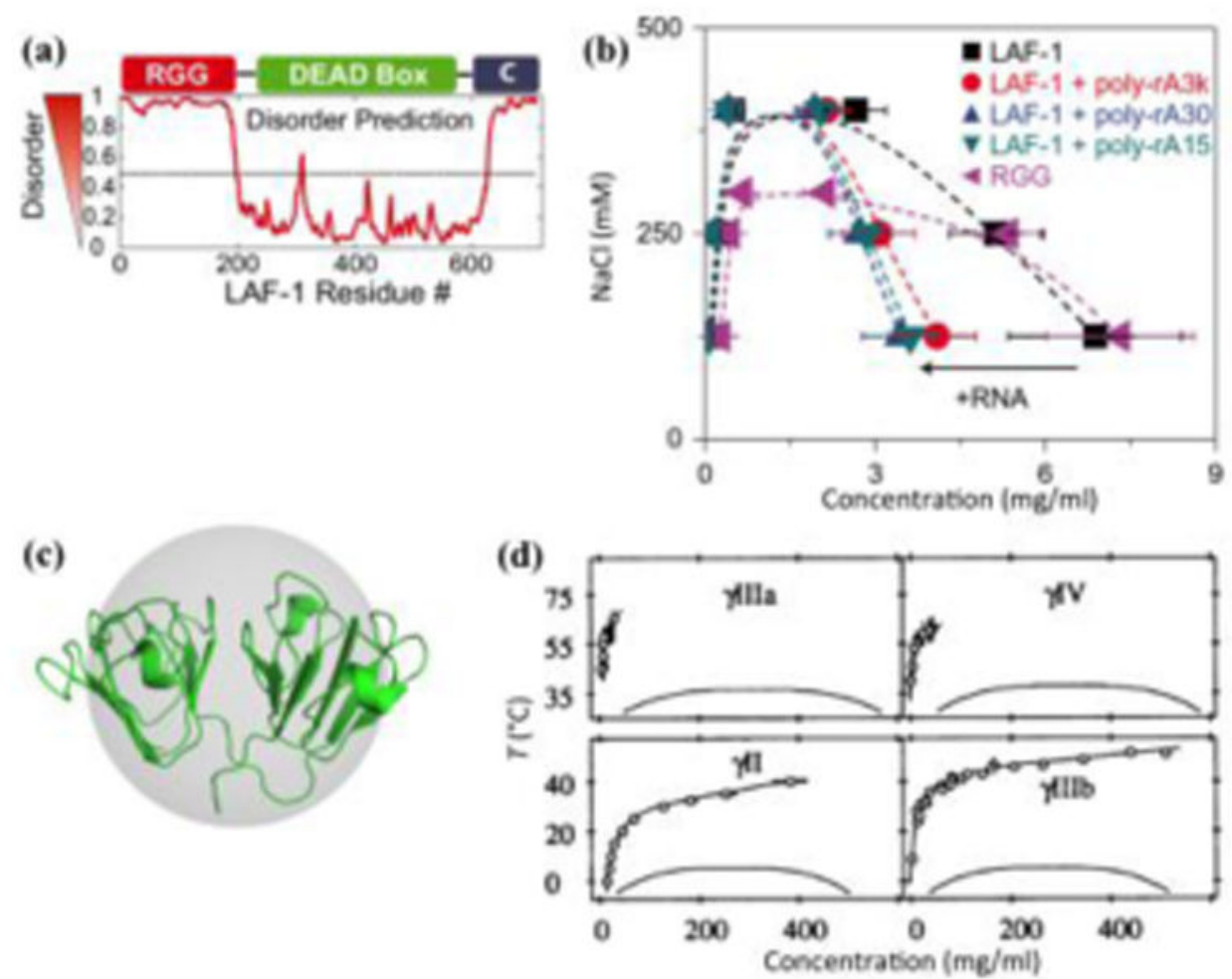

Figure 1.

Disparate phase diagrams of disordered and structured proteins. (a) LAF-1 consists of two terminal disordered regions and a structured domain. The $\mathrm{N}$-terminal disordered region (RGG) can bind RNA. Reproduced from ref [13]. (b) Liquid-liquid coexistence curves of LAF-1 and its disordered RGG region, in the presence and absence of polyadenylate RNA (poly-rA) of 15, 30, and 3000 nucleotides. A leftward arrow labeled with "+RNA" indicates the shift of the droplet branch in the presence of RNA. Reproduced from ref [29]. (c) Structure of $\gamma$ II-crystallin, embedded in a sphere to emphasize the globular shape. (d) Phase diagrams of 4 types of $\gamma$-crystallins, which are members of a highly homologous family of proteins. Symbols and the associated upper curves represent the fluid branch of the fluidsolid phase diagram, and lower curves are fits to liquid-liquid coexistence data from ref [36]. The fact that the liquid-liquid coexistence curve lies entirely below the fluid branch indicates metastability of the liquid-liquid phase separation relative to the fluid-solid phase separation (cf Box 3 Figure I). Reproduced from ref [78]. 


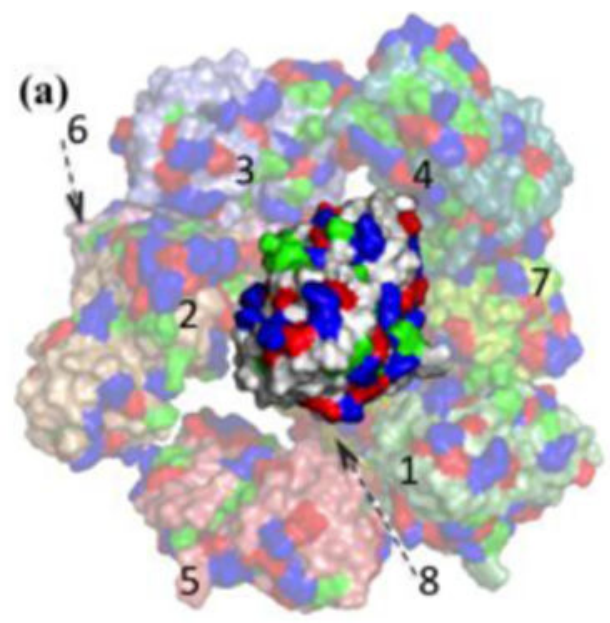

(b)

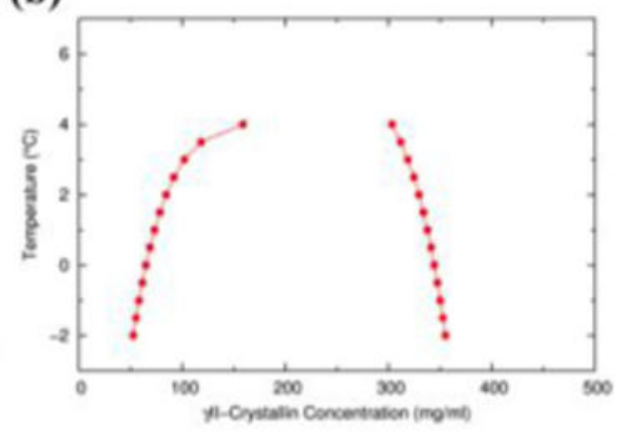

Figure 2.

Atomistic view of liquid-liquid phase separation (LLPS) for $\gamma$ II-crystallin. (a) Snapshot of a transient bonding network. The $\gamma \mathrm{II}$-crystallin molecule at the center presently is in contact with four other molecules (numbered 1-4); four molecules (numbered 5-8) further into the background and in contact with the bonding partners of the central molecule are also shown, but bonding partners of the central molecule in the foreground are not displayed. Molecules are displayed as surface, with acidic (Asp/Glu), basic (Arg/Lys), and hydrophobic (Leu/Ile/Val/Met/Phe) residues in blue, red, and green respectively. (b) Calculated LLPS coexistence curve for $\gamma$ II-crystallin. Reproduced from ref [46]. 

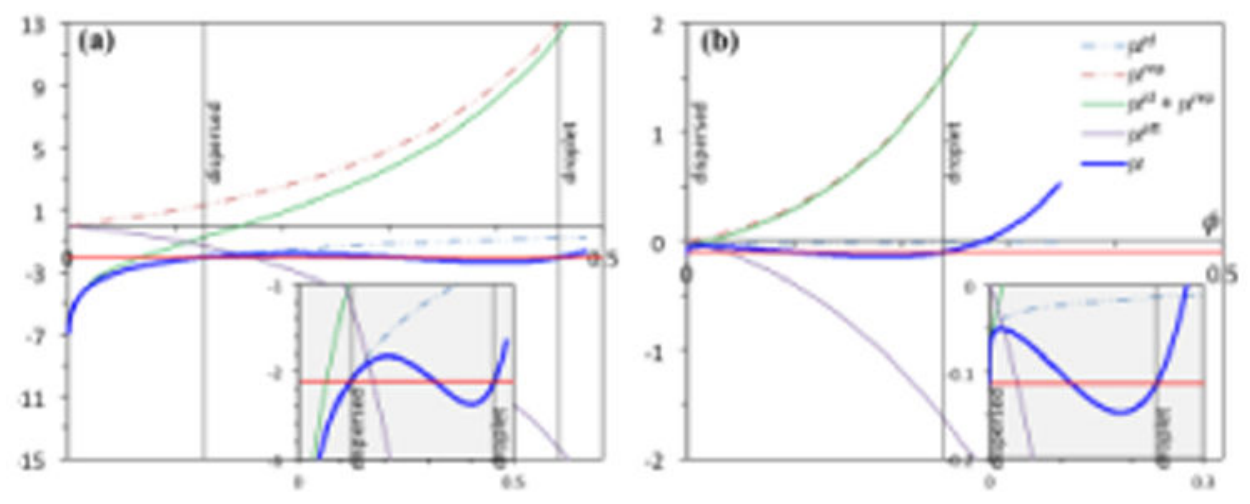

Figure 3. Key Figure

Decomposition of the chemical potentials of colloid and polymer models. (a) The chemical potential $(\mu)$ of a colloid suspension is given by Box 3 Equation [VI], with the ideal $\left(\mu^{\text {id }}\right.$; assuming $v_{\mathrm{Q}}=v_{\mathrm{p}}$ ), repulsive ( $\left.\mu^{\mathrm{rep}}\right)$, and attractive $\left(\mu^{\mathrm{rep}}\right)$ components, respectively, represented by the three terms on the right-hand side. Results for $\delta / \sigma=0.15$ and $k_{\mathrm{B}} T / \varepsilon=0.6$ are shown. (b) The chemical potential of a polymer solution is shown on a per monomer basis $(L=100) . \mu^{\mathrm{id}} / L=(1 / L) \ln \phi ; \mu^{\mathrm{rep}} / L$ is given by Box 4 Equation [V]; and $\mu^{\text {att }} / L$ is given by Box 4 Equations [VI] - [VIII] (for $\delta / d=0.5$ and $k_{\mathrm{B}} T / \varepsilon=2.4$ ). Abscissa: volume fraction $\phi$; ordinate: chemical potentials and their components in units of $k_{\mathrm{B}} T$. Horizontal red lines dissect the $\mu$ versus $\phi$ curves (blue) with equal areas; $\phi$ values for the dispersed and droplet phases are indicated by vertical black lines; the $\phi$ value for the dispersed phase of the polymer solution is so small that a vertical line cannot be distinguished from the ordinate axis. A view of the dissection, enlarged in the vertical direction, is shown as insets (gray shading). 


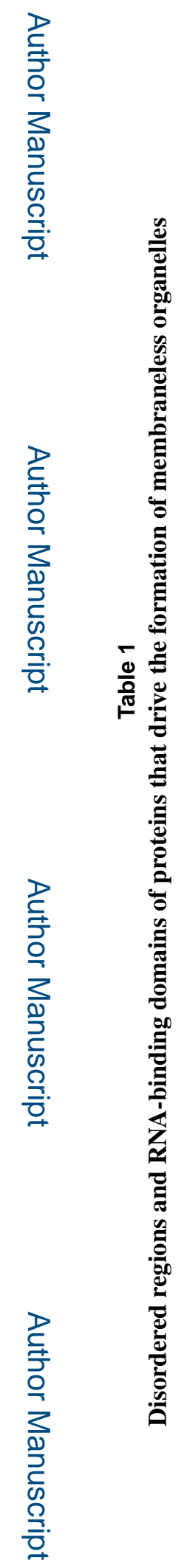

\begin{tabular}{|c|c|c|c|c|c|c|c|c|c|c|c|c|}
\hline 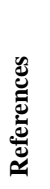 & $\begin{array}{l}\overline{\mathrm{N}} \\
\dot{\Xi}\end{array}$ & $\Xi$ & $\stackrel{\Xi}{\Xi}$ & 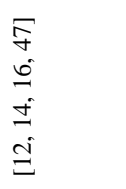 & $\Xi$ & $\Xi$ & $\begin{array}{l}\text { I } \\
\text { 品 }\end{array}$ & $\underline{n}$ & 寻 & $\begin{array}{l}\sqrt{n} \\
\dot{\Xi}\end{array}$ & $\begin{array}{l}\widetilde{N} \\
\stackrel{v}{=}\end{array}$ & $\begin{array}{l}\overline{\mathrm{O}} \\
\dot{\Xi}\end{array}$ \\
\hline 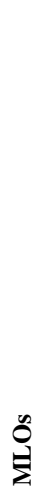 & 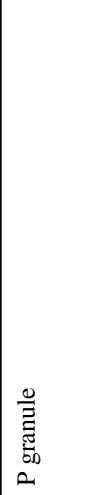 & $\begin{array}{l}\text { D } \\
\text { 茎 } \\
\bar{z}\end{array}$ & 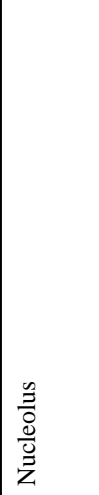 & 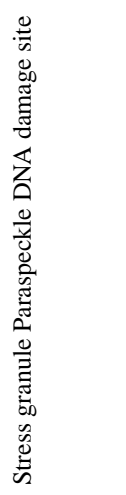 & 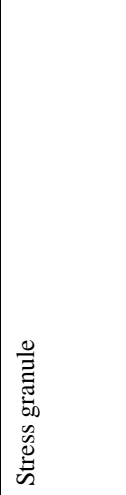 & $\begin{array}{l}\frac{3}{0} \\
0 \\
0\end{array}$ & \begin{tabular}{|l|}
$\frac{a}{0}$ \\
$\frac{0}{0}$ \\
$\frac{0}{0}$ \\
$z$ \\
$z$
\end{tabular} & 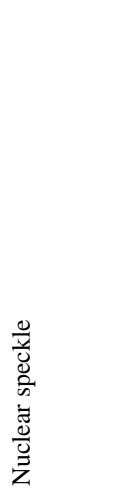 & 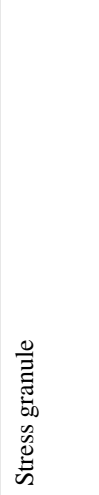 & 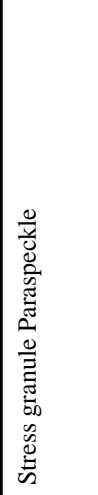 & 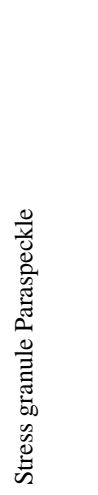 & 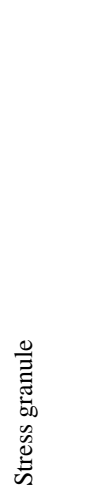 \\
\hline 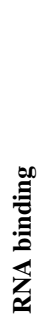 & 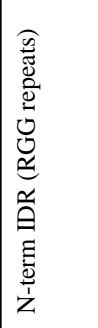 & 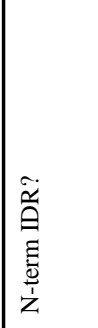 & 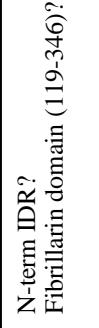 & 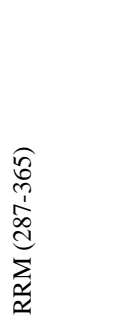 & 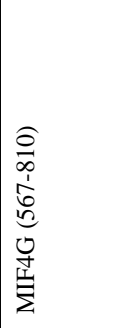 & 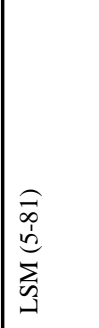 & 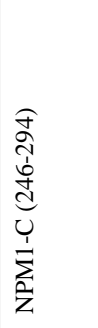 & 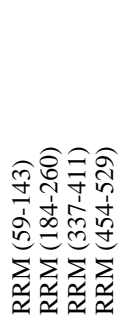 & 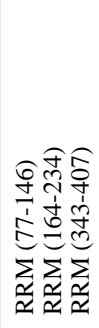 & 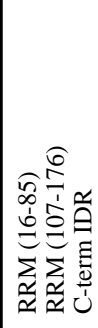 & 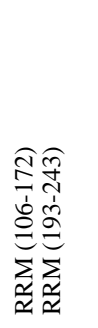 & 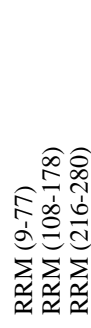 \\
\hline 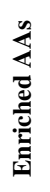 & 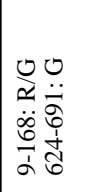 & $\begin{array}{l}0 \\
\ddot{\sim} \\
\tilde{i} \\
\infty \\
\infty\end{array}$ & 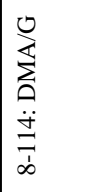 & 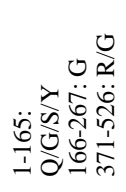 & 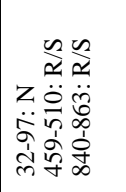 & & 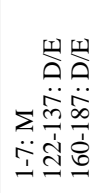 & 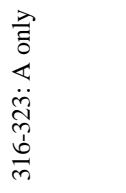 & 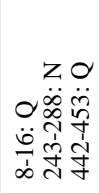 & $\begin{array}{l}0 \\
\dot{a} \\
\hat{a} \\
\dot{1} \\
\alpha \\
\end{array}$ & $\begin{array}{l}0 \\
\ddot{m} \\
\dot{y} \\
\stackrel{+}{+} \\
\stackrel{v}{v}\end{array}$ & \\
\hline है & 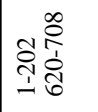 & 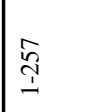 & $\stackrel{\Xi}{I}$ & 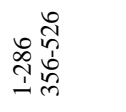 & 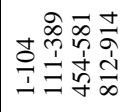 & $\begin{array}{l}\infty \\
\frac{\infty}{\infty} \\
\infty \\
\infty\end{array}$ & 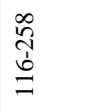 & & 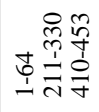 & 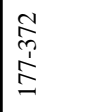 & $\begin{array}{l}\frac{\partial}{7} \\
\dot{0} \\
\infty \\
\end{array}$ & $\begin{array}{l}\infty \\
\stackrel{\infty}{\infty} \\
\stackrel{m}{n}\end{array}$ \\
\hline 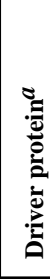 & 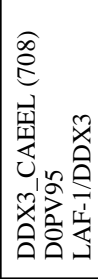 & 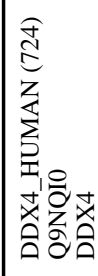 & 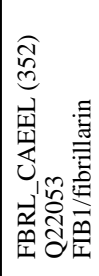 & 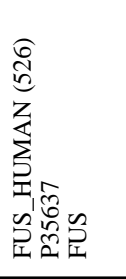 & 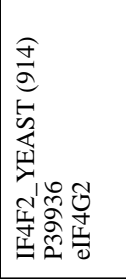 & 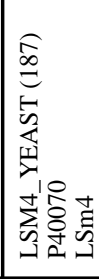 & 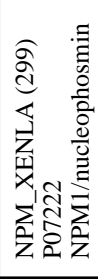 & 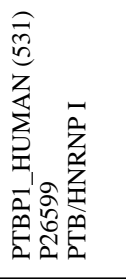 & 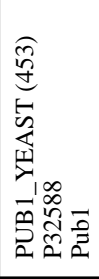 & 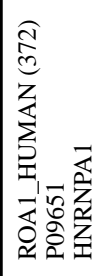 & 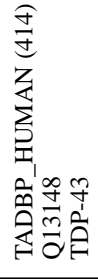 & 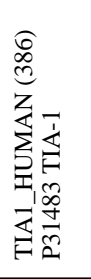 \\
\hline
\end{tabular}

Trends Biochem Sci. Author manuscript; available in PMC 2019 July 01. 


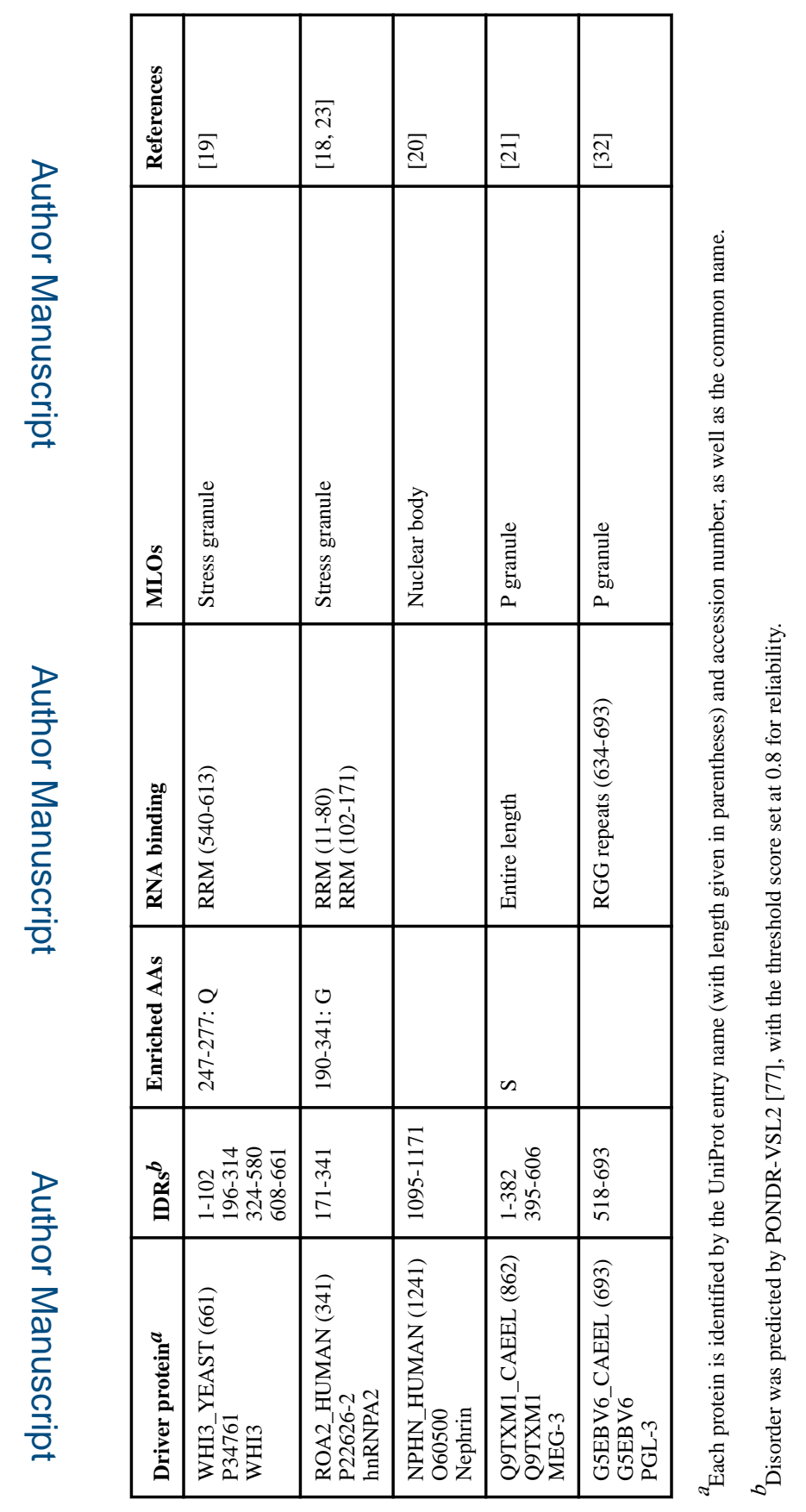

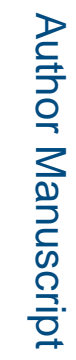

